\title{
Power transmission entropy
}

\author{
Serhan Ozdemir \\ Artificial Iytelligence and Design Laboratory, \\ Mechanical Engineering Department, \\ Izmir Institute of Technology, \\ 35437 Izmir, Turkey \\ E-mail: serhanozdemir@iyte.edu.tr
}

\begin{abstract}
Mechanical transmissions have been characterised traditionally by their transmission efficiencies. This is given by the ratio of the output to the input of the transmitted power. Unfortunately, the power transmission phenomenon is slightly more complex than that. As any designer would agree, each of these transmission localities is a source of uncertainty. Once formulated, this statement of uncertainty would reflect the designer's trust in the transmission. By virtue of the proposed approach, power transmission is no longer a deterministic entity but becomes a probabilistic one. This paper discusses the overlooked uncertainty inherent in every transmission.
\end{abstract}

Keywords: power transmission; efficiency; entropy; uncertainty; power divergence.

Reference to this paper should be made as follows: Ozdemir, S. (2008) 'Power transmission entropy', Int. J. Heavy Vehicle Systems, Vol. 15, No. 1, pp.82-88.

Biographical notes: Serhan Ozdemir was awarded a scholarship following his graduation from the Mechanical Engineering Department of Dokuz Eylul University, Izmir, Turkey. Having received his Masters Degree in 1996 from the Illinois Institute of Technology, he went on with his PhD studies at the University of Florida. On his return to Turkey, he founded the artificial intelligence and design laboratory at Izmir Institute of Technology. Even though the research at the laboratory ranges from intelligent control of artificial limbs to interpretation of EKG signals by fractals, the primary focus is on the processing of time series for machine health and fault diagnostics.

\section{Introduction}

Traditionally, mechanical transmissions have been characterised by their transmission efficiencies. This efficiency is given by the ratio of the power output to that of the input. Unfortunately, the power transmission phenomenon is slightly more complex than that. The simplification of the calculation of the power transfer has both up and downsides to it. This process of defining the power 'junction' by a constant of efficiency simplifies the computations and gives the designer an upper hand by allowing him to focus on more complicated matters. The downside to this simplification is that power transmission is a complicated matter itself. 
Each transmission of power or torque should be regarded in its own right. These junctions could safely be thought of as portals where an entity of two different magnitudes coexists. As any designer would agree, each of these portals is a source of uncertainty. Thus each transmission of power, or torque for that matter, should not only be designated by an efficiency but also by an expression of uncertainty. This statement of uncertainty would reflect the designers' trust in the transmission.

\section{Sources of uncertainty}

Practically every connection in a mechanical system is a source of entropy. The ones with the highest entropy are:

- Belt drives. Angular uncertainty due to slippage.

- Clutch mechanisms. Similar to belt drives.

- Chain drives. Variation in linear chain speed.

- Gear mesh. Minor uncertainty due to power loss and possible backlash, favoured.

For each and every one of these single drives, various entropies could be defined. For example, belt drives and clutches could be designated by a positional entropy, whereas a chain drive could be designated by a speed entropy etc. This is left to another study.

\section{Entropy as a measure of uncertainty}

Shannon, in his seminal work in 1948, has described a formula for uncertainty. He devised a way to account for the uncertainty of any received message, say, in bits. The idea was to quantify the possibility of error due to channel noise. Information theory is naturally beyond the scope of this work. Tutorials and texts are widely available on the matter. One good application of entropy to sensory data processing on navigation may be interesting in Fox et al. (1999).

A brief formulation of entropy is expressed below, Knessl (1998). The uncertainty by Shannon entropy was given by:

$$
H=-\sum_{i=1}^{M} P_{i} \log _{2} P_{i}
$$

where,

$$
\begin{array}{ll}
M: & \text { Number of symbols } \\
P_{i}: & \text { Probability of a symbol appearing } \\
H: & \text { Uncertainty. }
\end{array}
$$

Here, the base 2 logarithm refers to the two states of operation. The number of symbols is also limited to 2. These two symbols represent 'signal' and 'no signal' states. In this paper, they will mean 'power pass' and 'no pass' states. From now on, when a transmission is given by a constant of efficiency, this constant will be considered as the 
probability of power flow. Suppose that a unit has a probability of $\eta$ (efficiency) to transmit power, that is just another way of saying that the power will be absorbed with a probability of $(1-\eta)$.

\section{Entropy in power transmission}

Characterising a discontinuity for the power to flow in entropy terms will have the following characteristics:

- considers transmission as a probabilistic event

- $\quad$ penalises transmissions at low efficiency points more than basic algebra

- gives an analytical expression to optimise for the power to flow.

Transmission of power is indeed a probabilistic event. Since temperature, material characteristics etc., at contact surfaces all vary, so does the transmission efficiency. This makes the constant of efficiency a stochastic entity, and the transferred power a probabilistic phenomenon. Logarithmic formulation of uncertainty penalises low efficiencies further than simple algebraic operations would do. The last part is especially important when the power has more than one route to flow. This is true of hybrid transmissions, where motion is split and partly transmitted through, say, over a planetary gear set and partly through a pulley-belt mechanism. Such a performance measure would fit well its niche in an application such as Ozdemir and Schueller (2002). The percentage to follow through the gear set will be determined by an entropy minimisation process.

For a power line with power transmission localities, it would be proper to define a general 'Total Transmission Entropy' or TTE.

$$
\text { TTE }=-\sum_{k=1}^{B} \sum_{j=1}^{N} \sum_{i=1}^{2} P_{i} \log _{2} P_{i}
$$

where

$N$ : Total number of elements that can be assigned an efficiency of transmission on a single branch

$B$ : Total number of branches that power is transmitted through

$P_{i}$ : Transmitted power through a designated route.

In multi-path torque transmission, the logical idea would be to run most of the power along the most efficient line. The limiting factors could probably be strength, and/or design considerations. Obviously, for two routes of varying efficiencies, power should be split proportionally to the efficiencies.

TTE could be simplified with no loss of generality:

$$
\mathrm{TTE}=-\sum_{k=1}^{B} \sum_{i=1}^{N} P_{i} \log _{2} P_{i}
$$

where only the 'pass' state is considered, and the resultant of each branch is considered as the sum of the entropies of individual units along the path as in Figure 2. 


\section{Introduction of uncertainty as a design parameter}

As stated above, various mechanisms of power would affect the transmission in degrees depending upon the type of the mechanism. Assuming an exponential expression between the efficiency and the probability of transmitting power, one could define a relationship where the exponent $\alpha$ is always in the neighbourhood of one.

$$
\eta_{T}=\eta^{\alpha}
$$

where $1 \leq \alpha$, and, one would expect $\alpha \cong 1$ for gearsets, and slightly above for the rest (clutches, belt drives, etc.). In the remainder of this brief, $\eta_{T}$ would be designated as $\eta$ for simplicity.

An example might be given to demonstrate how successive units on the same branch increase uncertainty compared to a single one. Incidentally, this could be taken as an alternative definition of reliability.

Consider Figure 1. Here, take for instance, the efficiencies of the meshes at points $a$, $b$, and $c$ as $\eta_{a}=0.95, \eta_{b}=0.99$ and $\eta_{c}=0.97$, respectively. Also suppose this is a gear train. By the definition in equation (3), simply because there are more transmission localities in the path, this augments the uncertainty. The uncertainty in terms of the entropy is computed as follows:

Figure 1 A generic two-branch three-element power split scenario

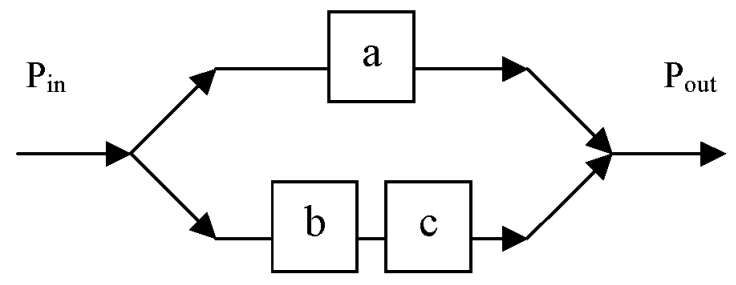

Individual entropies, the sum, and the entropy of the unit by the resultant efficiency are given in Table 1. Path (a) possesses the highest uncertainty as expected from the lower efficiency. Calculations at (b) and (c) juxtapose a contrast that only a slight difference in $\eta$ creates. This proves that logarithmic cost function indeed penalises more than mere comparison.

Table 1 Entropies at various localities

\begin{tabular}{lcccc}
\hline$E\left(\eta_{a}\right)$ & $E\left(\eta_{b}\right)$ & $E\left(\eta_{c}\right)$ & $E\left(\eta_{b} \eta_{c}\right)$ & $E\left(\eta_{b}\right)+E\left(\eta_{c}\right)$ \\
\hline 0.0703 & 0.0144 & 0.0426 & 0.0561 & 0.0570 \\
\hline
\end{tabular}

Please note that considering the lower branch as a single unit, and producing an efficiency as a multiplication in the usual way yields a value of 0.0561 , whereas adding individual entropies in the TTE way produces a higher uncertainty. This can be interpreted as follows: 
- TTE regards serial transmission junctions with reduced trust

- it suggests slightly more power be transferred from serial branches to single unit (less uncertain) ones

- it discerns among types of mechanisms.

\section{Optimal power distribution with the least uncertainty}

By integrating the uncertainty term in a cost function, a real time route optimisation could be scheduled. Consider, for example, the cost function:

$$
\operatorname{Min} J=F(p)-\sum_{i=1}^{B} \eta_{i} w_{i} P_{\text {in }} \log _{2}\left(\eta_{i} w_{i} P_{\text {in }}\right) .
$$

Subject to

- $S(w)$

- $\quad \sum_{i=1}^{B} w_{i}=1$.

where $F(p)$ could be the cost of running power through a certain path in, say, fuel, or amount of vibration etc., and $S(w)$ is scheduled power split for a power split transmission, and $w_{i}$ is the percentage of input power through $i$ th path.

Such a cost function would take into consideration the uncertainty as well as all the design requirements. By weighting these separate factors, by the order of importance or cost, an optimal power split policy may be obtained.

\section{Lower threshold of power split}

The output power for a $B$ branch system may be described by

$$
P_{\text {out }}=-P_{\text {in }} \sum_{i=1}^{B} \eta_{i} w_{i}
$$

Now let us consider again Figure 2. Supposing that power branches out in two, the output power could be written as:

$$
P_{\text {out }}=P_{\text {in }}\left(w_{1} \eta_{1}+w_{2} \eta_{2}\right)
$$

Figure 2 Simplified version where each branch is designated by an efficiency

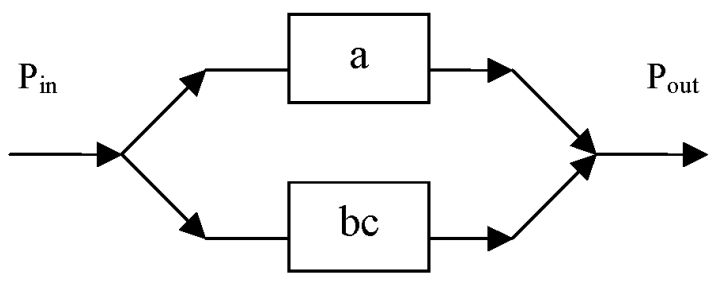


The TTE for this case is

$$
\mathrm{TTE}=-\left(w_{1} \eta_{1}\right) \log _{2}\left(w_{1} \eta_{1}\right)-\left[\left(1-w_{1}\right) \eta_{2} \log _{2}\left(\left(1-w_{1}\right) \eta_{2}\right)\right] .
$$

The worst case power split ratio can now be found by deriving TTE with respect to $w\left(w_{1}\right)$ and then setting this to zero. This value of $w_{1}$ will give the lower threshold of power split through the 1st path.

$$
\frac{\mathrm{d}(\mathrm{TTE})}{\mathrm{d} w_{1}}=0
$$

Hence,

$$
\eta_{2} \log _{2}\left[\left(1-w_{1}\right) \eta_{2}\right]-\eta_{1} \log _{2}\left(w_{1} \eta_{1}\right)+\eta_{2}-\eta_{1}=0 .
$$

Optimal power split ratio should remain well away from the worst case scenario found by the above formula.

$$
w_{\mathrm{opt}} \gg w_{1} \text {. }
$$

As an example case, suppose now that for the same two branch system, the first path has 0.95 and the second has 0.45 efficiency. Figure 3 shows that under these circumstances, the worst case appears around when $35 \%$ of power flows through the first route. This rate also represents the highest uncertainty. As more and more power is transmitted through line 1 , uncertainty approaches zero.

Figure 3 Total transmission entropy vs. $w_{1}$

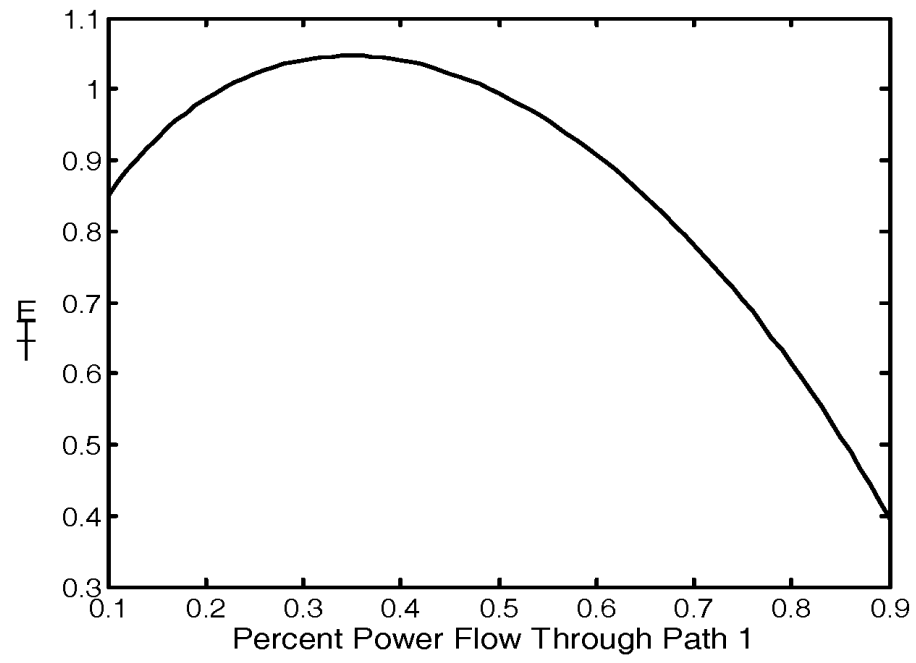

\section{Conclusions}

The classical design concept have always tended to ignore the transmission junctions and labelled them by a simple constant. That concept has also regarded the power transfer phenomenon as a deterministic event in a narrow simplistic view. However, power 
transfer is a highly stochastic and conditional property of the systems that perform this transmittal. Changing working conditions, imperfect manufacturing techniques, varying loads all affect the constant that distinguishes the locality. This study has presented a new approach in the design of mechanical transmissions. The idea of transmission entropy is the novelty of this paper, and, to the knowledge of the author, this is the first time it is used in literature in this context. This discussion is far from complete. A later work ought to deal with the conditional aspects of transmissions where varying performances of successive elements on a single line could influence the efficiency of the system. Also only Shannon entropy is utilised. The results could be compared with other entropy definitions.

\section{References}

Fox, D., Burgard, W. and Thrun, S. (1999) 'Markov localization for mobile robots in dynamic environments', J. Artificial Int. Research, Vol. 11, pp.391-427.

Knessl, C. (1998) 'Integral representations and asymptotic expansions for Shannon and Renyi entropies', Applied Mathematics Letters, Vol. 11, No. 2, pp.69-74.

Ozdemir, S. and Schueller, J. (2002) 'A new hybrid CVT design: CVPSTs', Heavy Vehicle Systems, A Series of the Int. J. Vehicle Design, Vol. 9, No. 4, pp.319-332. 